\title{
Computation of Compton profiles in a weak laser field
}

\author{
Schmider, Hartmut; H. Smith, Jr. Vedene
}

Published in:

Physical Review A

Link to article, DOI:

10.1103/PhysRevA.53.3295

Publication date:

1996

Document Version

Publisher's PDF, also known as Version of record

Link back to DTU Orbit

Citation (APA):

Schmider, H., \& H. Smith, J. V. (1996). Computation of Compton profiles in a weak laser field. Physical Review A, 53(5), 3295-3301. https://doi.org/10.1103/PhysRevA.53.3295

\section{General rights}

Copyright and moral rights for the publications made accessible in the public portal are retained by the authors and/or other copyright owners and it is a condition of accessing publications that users recognise and abide by the legal requirements associated with these rights.

- Users may download and print one copy of any publication from the public portal for the purpose of private study or research.

- You may not further distribute the material or use it for any profit-making activity or commercial gain

- You may freely distribute the URL identifying the publication in the public portal

If you believe that this document breaches copyright please contact us providing details, and we will remove access to the work immediately and investigate your claim 


\title{
Computation of Compton profiles in a weak laser field
}

\author{
Hartmut Schmider \\ Department of Chemistry, Chemical Physics, Technical University of Denmark, DTU-207, DK-2800 Lyngby, Denmark \\ Vedene H. Smith, Jr. \\ Department of Chemistry, Queen's University Kingston, Ontario, Canada K7L 3N6 \\ (Received 3 October 1995)
}

\begin{abstract}
A modified numerical scheme for the evaluation of Compton profiles in a weak laser field, based on an expression by Jain and Tzoar [Phys. Rev. A 18, 538 (1978)], is derived. The scheme is based on the reciprocal form factor and is computationally preferable to the original method. The scheme requires either a numerical Fourier transformation or the calculation of a one-dimensional integral, both of which are easily achieved. We focus on the numerical integration, which is readily performed with a Gauss-Chebyshev quadrature. Stable results may be obtained by avoiding numerical derivatives. Some numerical tests are performed to study the convergence properties of the old and modified methods. The modified scheme is applied to study some atomic and molecular systems. The dependence of the profile modulation on the atomic number $Z$ throughout the periodic table is established.

PACS number(s): 34.80.Qb, 32.10.-f, 33.15.-e
\end{abstract}

\section{INTRODUCTION}

The inelastic scattering of $\mathrm{x}$ rays from bound electrons may be described in the framework of the impulse approximation (IA) [1] by means of a projection of the singleparticle momentum density $\pi(\vec{p})$ onto a scattering vector that is determined by the geometry of the measurement. This projected quantity is called the Compton profile $[2,3]$ and plays a pivotal role in linking experimentally accessible scattering cross sections to quantum-mechanical observables:

$$
J\left(p_{z}\right)=\iint \pi\left(p_{x}, p_{y}, p_{z}\right) d p_{x} d p_{y} .
$$

If the scattering takes place in the presence of coherent electromagnetic radiation, for example, in a laser cavity, multiphoton absorption and emission processes will lead to a strong modulation of the profile. Jain and Tzoar [4] have derived a formula for the approximate calculation of such modulated Compton profiles for bound electrons. The approximations in their derivation include the aforementioned impulse approximation, i.e., the momentum transfer in the process is considered to be significantly higher than the typical momenta of the target electrons. Additionally, the intensity of the coherent field is supposed to be small enough to enable the neglect of the impact of the field on the Coulomb potential in which the bound electrons are moving. As a result, only the final state of the ejected electron (which is considered to be a plane wave in the IA) is assumed to be modified by the presence of the field. The resulting expression involves an infinite summation over the square of Bessel functions, which in general may not be performed analytically. However, a series expansion yields the approximate expression

$$
J^{L}(q)=\sum_{\nu=0}^{\infty} \frac{\alpha^{2 \nu}}{2^{2 \nu}(\nu !)^{2}} \frac{d^{2 \nu} J(q)}{d q^{2 \nu}}
$$

where $q$ denotes the absolute value of the momentum transfer, as the argument of the spherically averaged quantity. Equation (2) includes only even-ordered derivatives of the unmodulated profile $J(q)$, and a field parameter $\alpha$. The latter is a measure of the strength of the laser field, and is defined as [5]

$$
\alpha^{2}=e^{2} E_{L}^{2} / \hbar^{2} \omega_{L}^{2},
$$

with $E_{L}$ and $\omega_{L}$ denoting the electric-field amplitude and the frequency of the laser. Expression (2) requires the evaluation of derivatives, which may be analytically cumbersome, or numerically rather unstable. If the field parameter $\alpha$ is very small, only low-order derivatives are necessary, and it was pointed out [6] that in such cases the measurement of modulated profiles would supply information about the second derivative of the profile (and thus about the momentum density).

In the present paper, we recast Eq. (3) in order to arrive at a simpler way for the calculation of the modulated profile to full order, in which the evaluation of derivatives is avoided altogether.

\section{ALTERNATIVE EXPRESSION}

Let us consider the Fourier-cosine transform of the Compton profile (1) in a given direction,

$$
J(q)=\frac{1}{\pi} \int_{0}^{\infty} B(s) \cos (s q) d q
$$

The quantity $B(s)$ is called the reciprocal form factor [7], and it is of great use in the position-space interpretation of Compton profiles and momentum densities [7-10], because of its interpretation as an autocorrelation function of oneparticle wave functions describing the system $[7,8]$. If we insert Eq. (4) into (2), we obtain 


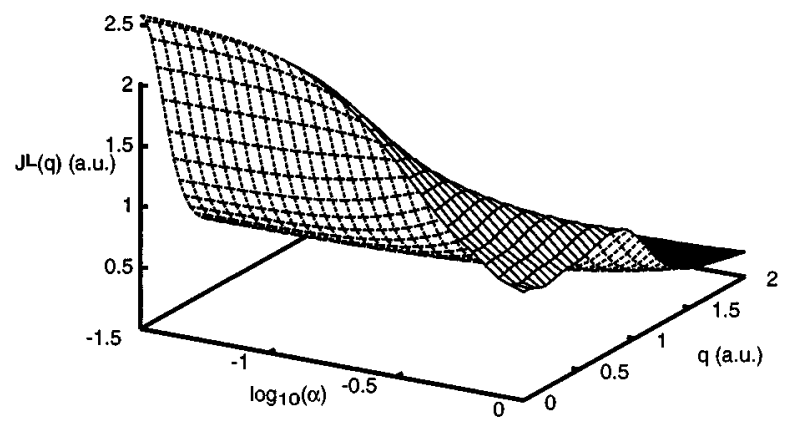

FIG. 1. Modulated Compton profile (CP) of the lithium atom. The CP is plotted as a function of the decadic logarithm of the field strength $\alpha$ and of the momentum transfer $q$. Note that the monotonicity of the unmodulated CP follows from its definition, but is not maintained for the modulated profile. The (atomic) units used are $e a_{0} \hbar^{-1}$.

$$
\begin{aligned}
J^{L}(q) & =\frac{1}{\pi} \int_{0}^{\infty} \sum_{\nu=0}^{\infty} \frac{(-1)^{\nu} \alpha^{2 \nu}}{2^{2 \nu}(\nu !)^{2}} s^{2 \nu} B(s) \cos (s q) d s \\
& =\frac{1}{\pi} \int_{0}^{\infty} M(\alpha, s) B(s) \cos (s q) d s,
\end{aligned}
$$

where we have abbreviated the infinite sum by $M(\alpha, s)$. The modulated reciprocal form factor $B^{L}(s)$ is therefore a product of the unmodulated one with a function $M(\alpha, s)$, which depends on $\alpha$. The function is a hypergeometric series, and may readily be identified as a zero-order Bessel function,

$$
M(\alpha, s)=J_{0}(\alpha s)
$$

The occurrence of the Bessel function is not surprising, since Bessel functions have served in the derivation of Eq. (2), and are of general importance in the calculation of modulated cross sections in scattering theory $[4,11,12]$. The Fouriercosine transform of $J_{0}$ is known;

$$
\int_{0}^{\infty} J_{0}(\alpha s) \cos (s q) d s=A(\alpha, q)=\left\{\begin{array}{ccc}
\frac{1}{\sqrt{\alpha^{2}-q^{2}}} & \text { if } & 0 \leqslant q<\alpha \\
\infty & \text { if } & q=\alpha \\
0 & \text { if } & \alpha<q .
\end{array}\right.
$$

The laser-modified Compton profile $J^{L}(q)$ is a convolution of the unmodified one $J(q)$ with this function $A(\alpha, q)$

$$
\begin{aligned}
J^{L}(q) & =\frac{1}{\pi} \int J_{0}(\alpha s) B(s) \cos (s q) d s \\
& =\frac{1}{2 \pi^{2}} \iiint A(\alpha, x) J(y) e^{i s[y-(x-q)]} d x d y d s \\
& =\frac{1}{\pi} \int A(\alpha, x) J(x-q) d x=\frac{1}{\pi} \int_{-1}^{1} \frac{J(q-\alpha z)}{\sqrt{1-z^{2}}} d z .
\end{aligned}
$$

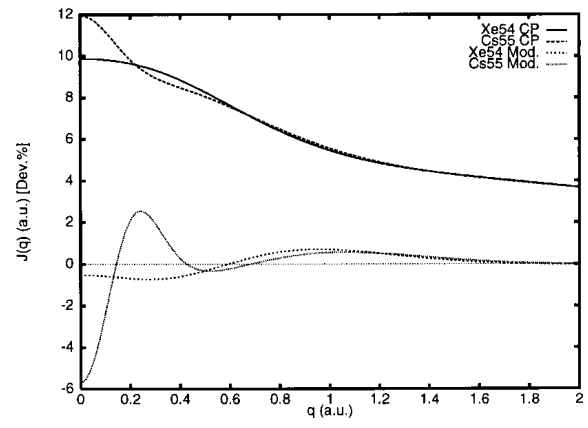

FIG. 2. Unmodulated Compton profiles and modulations in percent for the xenon and cesium atoms. The total profiles (full and dashed curves) are in (atomic) units of $e a_{0} \hbar^{-1}$, and normalized to the number of electrons. The modulations (dotted curves) are in percent. The fine-dotted horizontal line is a zero grid line.

The restriction of the function $A(\alpha, q)$ to a range of $2 \alpha$ and the symmetry of the Compton profile $J(q)$ permit the last step. The final integral in Eq. (8) is very easily evaluated with the use of a Gauss-Chebyshev quadrature, which has a weight function of $\left(1-z^{2}\right)^{-1 / 2}$.

We therefore propose the calculation of the laser-modified Compton profile by one of the following two routes:

(i) Explicit or numerical evaluation of $B(s)$ from the Compton profile. For experimental profiles, this may be done in a standard manner by fast Fourier transform methods. Multiplication by the Bessel function $J_{0}(\alpha s)$ and back transformation yields the desired modulated profile.

(ii) Numerical evaluation of the final integral in Eq. (8) by Gauss-Chebyshev quadrature. Points at the abscissas of that quadrature may be obtained explicitly for the theoretical case, or by interpolation from discrete data.

The second route is preferable in the case of the treatment of theoretical data. Often, only a few evaluations of the unmodulated profile are required. Higher derivatives do not explicitly enter the picture. For any given functional form of $J(q)$, the evaluation of the last integral in Eq. (8) is, of course, equivalent to the infinite series expansion in Eq. (2).

\section{SOME THEORETICAL EXAMPLES}

As expected for a convolution, the impact of a finite laserfield strength $\alpha$ on the Compton profile is a broadening. It was pointed out by Sharma et al. [6] that if the main contribution comes from the second derivative in Eq. (2) (as is the case if $\alpha$ is sufficiently small), there is exactly one point where the modulated and unmodulated profiles coincide: the inflection point of $J(q)$.

As the parameter $\alpha$ becomes larger, the neglect of higherorder terms in the series expansion (2) is not legitimate anymore, and the influence of the field is more complex. We consider here the example of the lithium atom in its restricted Hartree-Fock approximation (Fig. 1). The $x$ axis denotes the decadic logarithm of $\alpha$, whereas the $y$ axis is the momentum transfer $q$ in $\hbar a_{0}^{-1}$. As may be seen from the 

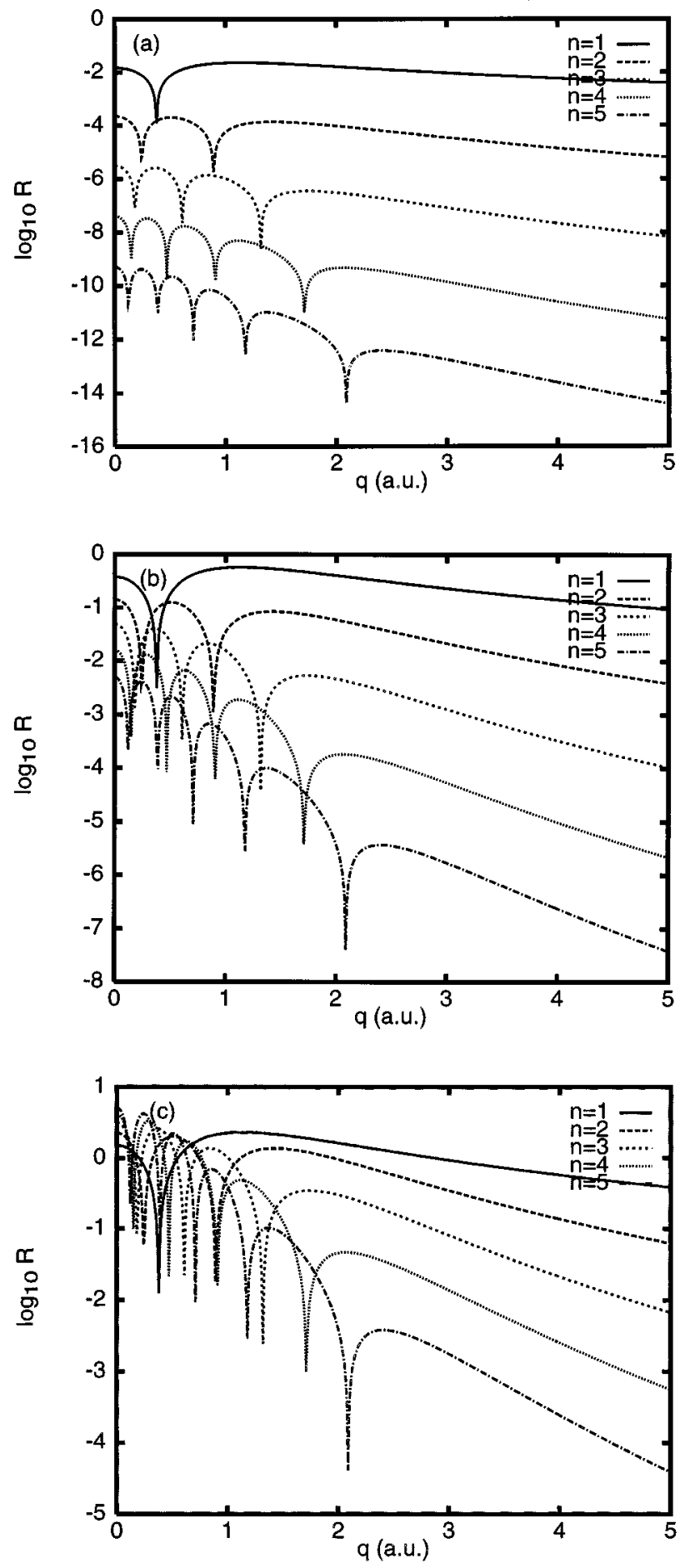

FIG. 3. Relative magnitude of the first five terms in expansion (2) for the hydrogen atom. Displayed is the decadic logarithm of the (absolute) ratio $R=\left|J^{n}(q)\right| / J(q)$, where $J^{n}$ denotes the term proportional to the $2 n$th derivative of the Compton profile. The different plots are for different field-strength parameters $\alpha$; (a) $\alpha=0.1$, (b) $\alpha=0.5$, (c) $\alpha=1.0$.

figure, for $\log _{10}(\alpha)>-1 / 2$, the convolution of the profile becomes so strong that the monotonicity of the Compton profile is destroyed. Instead, the modulated $J^{L}(q)$ exhibits a local minimum at $q=0$, with the maximum at increasingly large momenta. To calculate $J^{L}(q)$, one would have to include at least the fourth derivative of $J(q)$ for $\alpha$ 's in the

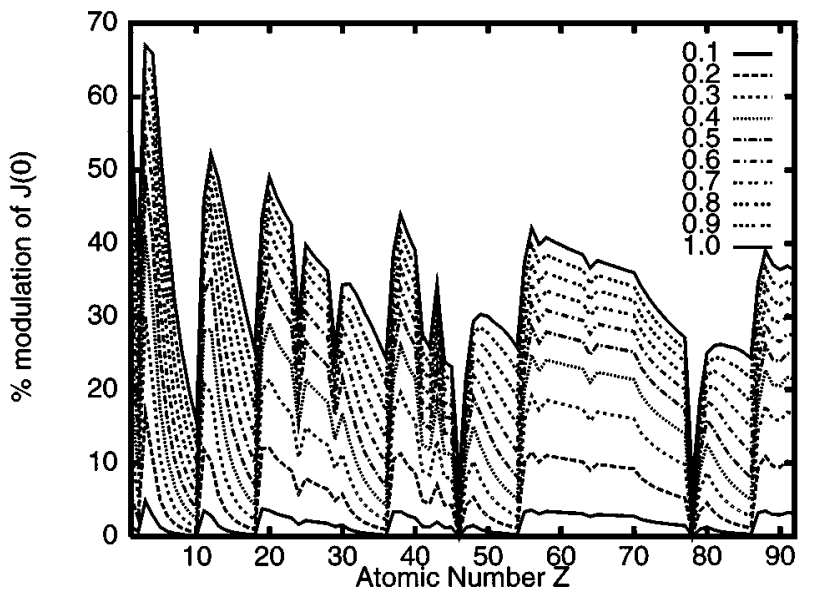

FIG. 4. Percent Modulation of the peak value of the Compton profile for the atoms in the periodic table (in the nonrelativistic Hartree-Fock approximation [14,15]). The different curves correspond to different values of the field-strength parameter $\alpha$. Note that the modulation is in all cases negative.

vicinity of 1 , which is admittedly a large value. "Reasonable" values are of the order of 0.1 , and values of $\alpha$ which are too high risk not satisfying the approximations made in the derivation of the basic expression (2).

The influence of the coherent light on the profile may differ greatly from system to system. Figure 2 shows the total unmodulated profiles, as well as the relative differences between modulated and unmodulated profiles of the xenon and the cesium atoms for the small-momentum range. The field strength parameter is 0.1357 a.u., which corresponds to a neodymium laser with an amplitude of $3 \times 10^{7} \mathrm{~V} / \mathrm{cm}$. Although the two atoms differ by only one electron, the influence of the laser is quite different: Whereas the "flat" profile of Xe (full line) is hardly affected, the more peaked one of Cs (long-dashed line) shows modulations of more than $5 \%$ (dotted line).

To gain experience in the importance of higher-order terms in the series expansion, the example of the hydrogen atom was considered. In this case, the Compton profile is proportional to $\left(1+q^{2}\right)^{-3}$, and the derivatives are easy to evaluate, albeit somewhat cumbersome. In Figs. 3, we have computed the ratio $\left|J^{n}(q)\right| / J(q)$, where $J^{n}(q)$ denotes the $n$ th-order term in the series expansion, i.e., the term proportional to the $(2 n)$ th derivative of $J(q)$. The $x$ axis is the momentum transfer, and the $y$ axis shows the decadic logarithm of the ratio. The different plots correspond to increasing values of $\alpha$. Note that there are $\nu$ "spikes" for each of the curves, arising from zeroes in the higher-order derivatives.

For $\alpha=0.1$ [Fig. 3(a)], terms with $n>1$ are certainly not of any great importance. Second-order contributions are of the order of $1 / 100 \%$, each term being approximately two orders of magnitude smaller than the previous one. Note that the modulation in this case is only in the $1 \%$ area to begin with. However, if $\alpha=0.5$ [Fig. 3(b)], the modulation is already almost as large as the profile itself for some momenta, and the second- and third-order terms reach values of several percent. The latter depend on the sixth derivative of $J(q)$ and are certainly not readily calculated for the general case, or 

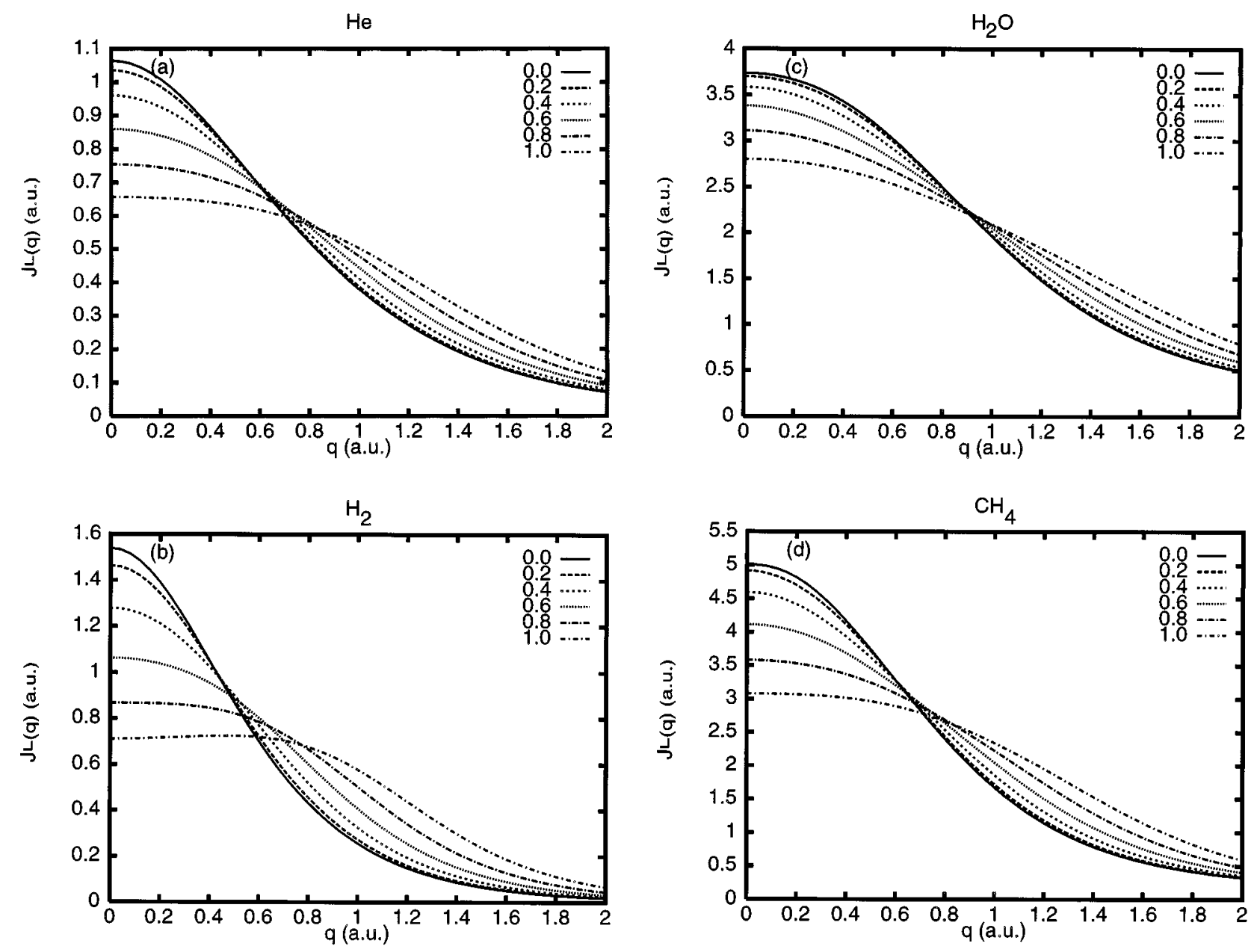

FIG. 5. Modulated spherically averaged Compton profiles based on experimentally determined unmodulated ones. The different curves correspond to different field-strength parameters (see legend). The profiles are represented by Lorentzian fits (see text). (a) helium, (b) hydrogen, (c) water, (d) methane. Atomic units $\left(e a_{0} \hbar^{-1}\right)$ are used.

even for discrete data. For $\alpha=1.0$ [Fig. 3(c)], all contributions up to fifth order (tenth derivative) are of similar importance for small $q$, and all larger than the profile itself. No calculation of the modulated profile on the basis of Eq. (2) has any prospect of success in this case. However, integral (8) is still simple to evaluate (e.g., at $q=0$ with a ten-point quadrature to an accuracy of $10^{-4} \%$; see Fig. 6).

\section{ATOMS IN THE PERIODIC TABLE}

The impact of a weak coherent field may be considered a measure of how "flat" the Compton profile is at any point. The degree of influence of each derivative on this quantity is a function of $\alpha$. As could be seen in the example of the hydrogen atom, the contributions are of similar size, but alternating sign, if $\alpha$ is large. For small $\alpha$, the second derivative is the main influence. At the peak, $J^{\prime \prime}(0)$, the second derivative is half the peak value [13] of the momentum density $\pi(0)$, so that in first order the modulation of the profile peak by a laser field is a measure for the number of electrons at rest.

We have used our integral scheme to calculate the modulations of the Compton peak height for the atoms of the periodic table. We use wave functions of the nonrelativistic relativistic Hartree-Fock type $[14,15]$ for the elements hydrogen to uranium to compute modulated peak values with $\alpha$ 's ranging from 0.1 to 1.0 . The relative modulations in percent are given in Fig. 4 as a function of the atomic number $Z$.

The obtained pattern mirrors the periodicity of the elements quite nicely: The noble gases $(Z=2,10,18,36,54,86)$ are invariably minima, arising from the fact that they exhibit the lowest momentum densities at $p=0$. There are two other cases of extremely low modulations: palladium and platinum, both with unoccupied $s$ shells in the valence. The maxima are usually exhibited by the alkali metals or earth-alkali metals $(Z=3,11,19,37,55,87$ or $Z=4,12,20,38,56,88)$. These atoms have occupied diffuse $s$ orbitals in the valence, which contribute to the momentum density in the form of strong peaks at zero momentum. As the atomic number increases throughout the shell, the valence $s$ orbitals spread out in momentum space, and the value of $\pi$ at the origin decreases. The laser modulation of $J(0)$ follows. With increasing fields, some details in the behavior through the periodic table change. For example, the alkali metals seem to be maximally influenced for low $\alpha$ 's and the earth alkali metals for larger ones. But the general picture remains the same throughout, and only the magnitude increases. Generally, lighter elements seem to be more 

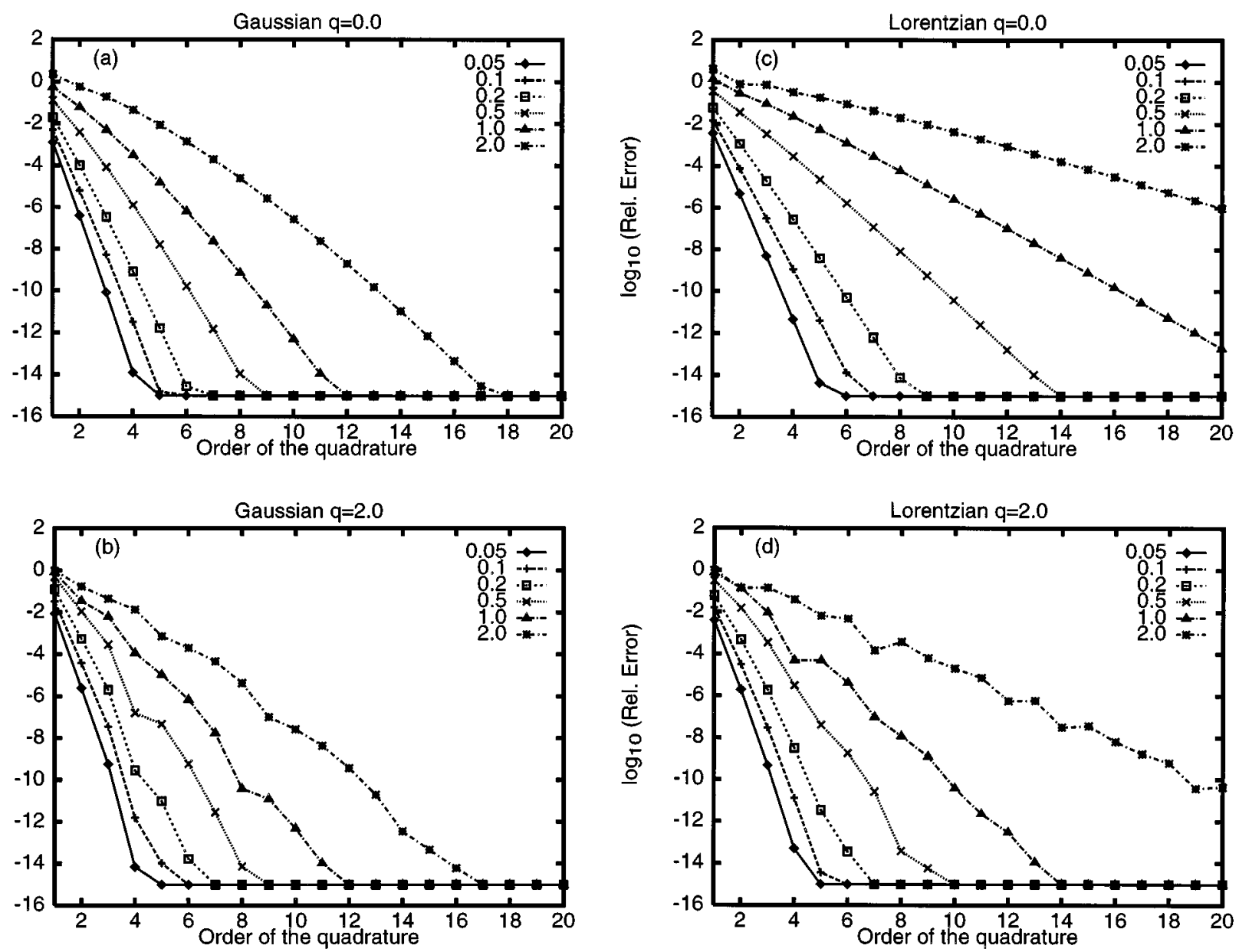

FIG. 6. Logarithm to the base 10 of the relative accuracy of the modulated profile as a function of the order of the Gauss-Chebyshev quadrature employed. The numbers on the $y$ axis are an approximate measure for the number of correct significant figures obtainable from numerical quadrature. The different curves denote different field-strength parameters $\alpha$. (a) and (b) correspond to Gaussian line shapes at $q=0$ and $q=2 \hbar a_{0}^{-1}$; (c) and (d) are the same for a hydrogenic Lorentzian.

strongly affected (up to almost $70 \%$ ) than heavier ones (around $30-40 \%$ for $\alpha=1$ ). Other local minima and maxima may usually be explained on the grounds of the electron configuration.

\section{V. “EXPERIMENTAL” PROFILES}

The treatment of experimental profiles requires some specific considerations. Since the data are discrete, derivatives as needed in Eq. (2) are not readily available. Interpolation of the data is usually done linearly to avoid error correlation, and second derivatives may not be obtained that way. If cubic splines are used for interpolation, only second derivatives are available, and the function exhibits discontinuities in the third derivative. It is, however, always possible to approximately evaluate the integral above, even if only linear interpolation is used. The results are of course not "exact" in either case.

In the practical application, the most likely strategy would be to build the multiplication by the Bessel function [see Eq. (5)] into the standard calculation of Fourier transforms of the experimental profile (e.g., for purposes of filtering),

$$
B^{L}(s)=2 \int_{0}^{\infty} J^{L}(q) \cos (s q) d q=J_{0}(\alpha s) B(s)
$$

We have chosen a simpler way to calculate some modulated profiles from experimental ones, by employing least-squares fits to a simple functional form from the literature. The cases presented here are sums of up to five terms of the form $a_{n} /\left[1+\left(q / \xi_{n}\right)^{2}\right]^{-2 n-1}$, where $a_{n}$ and $\xi_{n}$ are linear and nonlinear least-squares parameters, respectively. The fits employed here are for the systems $\mathrm{He}$ and $\mathrm{H}_{2}$ [16] [Figs. 5(a) and 5(b)], $\mathrm{H}_{2} \mathrm{O}[17,18]$ [Fig. 5(c)], and $\mathrm{CH}_{4}$ [19] [Fig. 5(d)]. The treatment, based on Gauss-Chebyshev integration, is, of course, equivalent to the explicit evaluation of derivatives of these functional forms, and the results are only as accurate as the fits on which they are based.

Figures 5 display the profiles obtained by variation of the parameter $\alpha$ in a range between 0 and 1 in steps of 0.2 . In all cases, the profiles becomes broader as $\alpha$ increases. The degree to which the intersection between the unmodulated and modulated profiles spreads in $q$ is a measure for the importance of higher-order terms in the series expansion. For small $\alpha$, the modulation may be expected to be dominated by the second derivative, and for the water system [Fig. 5(c)] all 
curves pass through more or less one point. This means that for water, the approximation by the first term would be fairly appropriate. For the other systems, however, this is not the case. Especially $\mathrm{H}_{2}$ exhibits a wide spread of the intersection points, and $J(q)$ even loses its monotonicity for larger $\alpha$. However, the starting approximation of a weak laser field is not satisfied anymore in those cases.

\section{NUMERICAL CONSIDERATIONS: CONVERGENCE}

To obtain information about the accuracy of the present method and its numerical reliability, we used two test functions for a convergence study. The first one was a simple unnormalized Gauss function $J(q)=e^{-q^{2}}$. For this, derivatives to arbitrary order are readily available in the form of the Hermite polynomials, and therefore the convergence of the original equation (2) to the correct value [calculated employing Eq. (8)] may be studied.

As expected, for small values of $\alpha \approx 0.1$, the modulation is dominated by the second derivative, and four significant figures are obtained already from the first term (proportional to the second derivative). Machine accuracy (double precision) requires only six terms. However, as $\alpha$ increases ( $\alpha \approx 0.5)$, six terms can only produce a single-precision agreement, and with even larger (and unrealistic) $\alpha$ 's (of the order of 1.0), single-precision agreement requires ten terms, i.e., the 20th derivative. For general cases, even the fourth derivative, which is necessary for realistic $\alpha$ values, is cumbersome to evaluate analytically, and inaccurate numerically.

Our integration scheme [Eq. (8)] may be checked on the same test system. We have done so by stepwise increasing the order of the Chebyshev quadrature, until the difference between two consecutive calculations was a double-precision zero. The resulting value was used as reference, and the relative errors of the previous calculations were computed. Their decadic logarithms are given in Figs. 6 as functions of the quadrature order. Errors smaller than $10^{-15}$ were artificially put to $10^{-15}$, which was assumed to be the limit of arithmetic accuracy in REAL*8. For the peak value $q=0$, the convergence is very smooth and very fast. Even for $\alpha=1.0$, a 12-point quadrature produces machine-accuracy. A pathological value such as $\alpha=2$ may be treated with 18 points. The same holds for $q=2$, although the convergence is slightly more erratic. Realistic values of $\alpha$ can be treated with ten-point quadratures without loss of accuracy.

Since a Gaussian line shape is not particularly realistic, we performed the same convergence test for the function $J(q)=\left(1+q^{2}\right)^{-3}$, which is proportional to the profile of the hydrogen atom. The results are shown in Figs. 6(c) and 6(d). The convergence is noticeably slower, particularly for high values of $\alpha$. For the peak value at $\alpha=1$, a 20-point quadrature only achieves 13 digits of accuracy, and for $\alpha=2$, only six significant figures may be obtained. For realistic values, however, a ten-point quadrature still achieves machine accuracy. For $q=2$, the convergence is quicker, but less smooth; again, a ten-point quadrature should serve the requirements for most cases.

\section{CONCLUSIONS}

A convolution integral on a finite range may replace an existing approximate expression for the laser-modulated
Compton profile in terms of even-ordered derivatives. The modified expression is both quickly convergent if treated with a Gauss-Chebyshev quadrature, and numerically stable, and therefore preferable for the treatment of discrete data and in cases where the analytical evaluation of derivatives is cumbersome and expensive.

The modulation of theoretical Compton profiles with varying strength of the coherent field was studied for some systems, and its dependence on the atomic number $Z$ through the periodic table was established. It was shown that systems with newly (half) filled $s$-valence shells show particularly strong modulation, whereas rare gases and the transition metals palladium and platinum are only very weakly affected. Experimentally derived fits were used to arrive at modulated Compton profiles that could be directly compared with (up to now not available) experimental data.

Finally, a few remarks about the validity of the approximations made in expression (2), and its impact on the application of the equivalent form (8) are in order. The formula is, in addition to the impulse approximation, based on the assumption of a homogeneous electric field on the spatial and temporal scale of the scattering event. Furthermore any influence of the coherent light on the target system is neglected, and only the modification of the final plane-wave state is taken into consideration. These assumptions require a field of low intensity, and are made even more severe by the exclusion of higher than first-order effects in the field frequency at a later point in the derivation [see Eqs. (18) and (19) in [4]]. With all this kept in mind, one might question if a calculation of the modulated profile to full order is desirable at all. As we have seen, if the field is very weak, higherorder terms are hardly noticeable [see, for example, Fig. 3(a)], and certainly outside the precision of even modern experiments (which should lie in the range of fractions of a percent in favorable cases). Even the first-order terms are small enough to warrant some suspicion about the relative size of the error inherent in the starting approximations. For larger values of the field-strength parameter, we obtain significant contributions from higher-order terms [e.g., Fig.3(b)], but the validities of the Taylor-series expansion and the series truncation in [4] are questionable at best. How questionable can ultimately only be assessed experimentally. We can speculate about an intermediate range where higherorder terms play a role, but the Jain-Tzoar approximation may still be used. However, a quantitative study of this question is outside the scope of this paper. It is not our intent to question (or confirm) the validity of the approximations made in Eqs. (2) and therefore (8). However, we feel that if such an expression is used for calculation, its truncation (in addition to all the approximations invoked already) should be avoided. It is certainly more elegant and convenient to evaluate it to full order with less computational effort than it takes to calculate only its first-order term directly. Its physical relevance is not affected by this in either a positive or negative sense.

\section{ACKNOWLEDGMENT}

This work was done with the financial support of the Natural Science and Engineering Research Council of Canada. 
[1] P. Eisenberger and P. M. Platzman, Phys. Rev. A 2, 415 (1970).

[2] Compton Scattering, edited by B. Williams (McGraw-Hill, New York, 1977).

[3] M. J. Cooper, Rep. Prog. Phys. 48, 415 (1985).

[4] M. Jain and N. Tzoar, Phys. Rev. A 18, 538 (1978).

[5] Our discussion is in terms of Compton profile and we follow the notation of Ref. [6]; we note that the original discussion in Ref. [4] was in terms of the double-differential cross section.

[6] B. S. Sharma, G. S. Singh, and A. N. Tripathi, J. Phys. B 14, 979 (1981).

[7] W. Weyrich, Habilitationsschrift, Technische Hochschule Darmstadt, 1978.

[8] R. Benesch, S. R. Singh, and V. H. Smith, Jr., Chem. Phys. Lett. 10, 151 (1971).

[9] F. M. Mueller, Bull. Am. Phys. Soc. 19, 200 (1974).

[10] P. W. Thulstrup, J. Chem. Phys. 65, 3386 (1976).
[11] L. Rosenberg, Adv. At. Mol. Phys. 18, 1 (1982).

[12] J. Z. Kaminski, Phys. Scr. 34, 770 (1986).

[13] The origin in momentum space is not necessarily a "peak" in an atomic momentum density. For many atoms, it is, in fact, a minimum (see, e.g., W. M. Westgate, A. M. Simas, and V. H. Smith, Jr., J. Chem. Phys. 83, 4054 (1985).

[14] C. F. Bunge, J. Barrientos, and A. V. Bunge, A. Data Nucl. Data Tables 53, 113 (1993).

[15] A. D. McLean and R. S. McLean, A. Data Nucl. Data Tables 6, 197 (1981).

[16] J. S. Lee, J. Chem. Phys. 66, 4906 (1977).

[17] A. C. Tanner and I. R. Epstein, J. Chem. Phys. 61, 4251 (1974), data from W. A. Reed, tabulated in this article.

[18] S. R. Gadre and P. K. Pathak, J. Chem. Phys. 92, 4327 (1990).

[19] R. W. Klapthor and J. S. Lee, Chem. Phys. Lett. 45, 513 (1977). 